\title{
1 Suturing of the Arachnoid Membrane for Reconstruction of the Cisterna Magna:
}

\section{Technical Considerations}

3

4 Umit Eroglu, MD, ${ }^{1}$ (orcid 0000-0001-8623-071X), Murat Büyüktepe, MD, ${ }^{1}$ Murat Zaimoğlu, 5 MD, ${ }^{1}$ Gokmen Kahilogullari, MD,${ }^{1}$ Hasan Caglar Ugur, MD, ${ }^{1}$ Mustafa Ağahan Ünlü, MD, ${ }^{1}$

6 Aaron Cohen-Gadol, MD, MSc, MBA ${ }^{2,3}$ (orcid 0000-0002-4946-1524)

7

$8{ }^{1}$ Department of Neurosurgery, Ankara University, School of Medicine, Ankara, Turkey; ${ }^{2}$ The

9 Neurosurgical Atlas, Carmel, Indiana; and ${ }^{3}$ Department of Neurological Surgery, Indiana

10 University, Indianapolis, Indiana

12 Correspondence: Aaron Cohen-Gadol, MD, MSc, MBA, Indiana University, Department of

13 Neurological Surgery, 355 W 16th Street, Suite 5100, Indianapolis, IN 46202;

14 cohen@nsatlas.com.

Short Title: Suturing of the Arachnoid Membrane

17

18 Key words: arachnoid membrane; cerebrospinal fluid leak; cisterna magna; dural closure; running-suture technique

21 This manuscript (i) is a unique submission, (ii) has not been submitted and is not being

22 considered for publication by any other source in any medium, and (iii) has not been published,

23 in part or in full, in any form.

Conflict of Interest: The authors have no personal, financial, or institutional interest in any of 26 the drugs, materials, or devices described in this article.

Disclosure of Funding: None.

29

Abbreviation: CSF, cerebrospinal fluid

This is the author's manuscript of the article published in final edited form as:

Eroglu, U., Büyüktepe, M., Zaimoğlu, M., Kahilogullari, G., Ugur, H. C., Ünlü, M. A., \& Cohen-Gadol, A. (2021). Suturing of the Arachnoid Membrane for Reconstruction of the Cisterna Magna: Technical Considerations. World Neurosurgery, 154, e724-e728. https://doi.org/10.1016/j.wneu.2021.07.129 
1 Suturing of the Arachnoid Membrane for Reconstruction of the Cisterna Magna:

2 Technical Considerations 


\section{ABSTRACT}

Background: Postoperative cerebrospinal fluid (CSF) fistula following cranial or spinal surgery is associated with increased morbidity and mortality. To prevent CSF fistulas, various techniques have been described.

Objective: To describe the arachnoid membrane continuous-running-suture technique in cisterna magna reconstruction for preventing postoperative CSF leakage.

Methods: After craniotomy and dural opening, the incision of the arachnoid of the cisterna magna was performed using a diamond blade. To prevent the arachnoid from drying out and shrinking during surgery, it was periodically irrigated with warm saline solution. Posterior fossa surgery was performed. While closing the membranes, the arachnoid membrane was closed with the running-suture technique. After the first surgical knot was made in the cranial end of the arachnoid opening, continuous suturing with a 2-mm distance between the stitches was performed without stretching them. After every 3 stitches, the free end of the thread was pulled gently along the suturing axis, and the edges of the arachnoid were closed. After the arachnoid edges were approximated, the surgical knot was tied. Watertight closure was checked by performing the valsalva maneuver at the end of the surgery.

Results: No CSF leakages were observed after surgery.

Conclusion: Arachnoid membrane suturing seems to be safe and effective in preventing postoperative CSF leakage and CSF-related complications. Using continuous running suturing alone, without any sealant, might be effective in cases with untraumatized arachnoid membrane. 


\section{BACKGROUND AND AIM}

Postoperative cerebrospinal fluid (CSF) fistula after cranial or spinal surgery is associated with increased morbidity and mortality. The incidence of postoperative CSF leakage has been reported as high as $32 \%$ after nontraumatic posterior fossa surgery. ${ }^{1}$ Postoperative CSF fistula can be complicated by meningitis, encephalitis, low-pressure headaches, chronic subdural hematomas and effusions, pseudomeningocele, arachnoiditis, dural-cutaneous fistula, pain, and pneumocephalus. ${ }^{2,3}$ The development of complications may depend on the location and size of craniotomy, as well as patient-related factors such as age and systemic disease. These complications often lead to prolonged hospitalization, reoperation, and increased mortality and morbidity rates. ${ }^{2}$ Various techniques to prevent CSF-related postoperative complications have been described. First, tight and reliable closure of the dura mater and fascia should be achieved. $^{3}$ Also, compressive bandaging, direct aspiration of subcutaneous CSF or diversion of ventricular CSF, ${ }^{4}$ skin sutures, various tissue adhesives,,${ }^{1,5}$ dural grafts, ${ }^{6}$ and surgical closure of the fistula are some of the interventions that can prevent and treat CSF leakage outside the closed dural incision. ${ }^{7}$

In this technical note, we report a case series in which continuous running suturing of the arachnoid membrane was included in cisterna magna reconstruction to prevent postoperative CSF-related complications.

\section{SURGICAL TECHNIQUE}

All surgeries were performed by the same surgeons. This case series included 11 patients without any history of previous surgery, and none of them had received radiotherapy treatment. All of the patients underwent surgery via a midline suboccipital approach. 
After the induction of general anesthesia, each patient was positioned in the prone position with head flexed using a three-point rigid cranial fixation system. After median skin incision, a suboccipital craniotomy or craniectomy was performed over the cerebellar hemispheres to expose the foramen magnum. The edges of the foramen were expanded by using a rongeur. While performing the craniotomy, dural injury was avoided, and no CSF leakage was observed. The posterior fossa dura was opened in the midline in a Y-shaped fashion over the cerebellar hemispheres bilaterally with a no. 15 blade. While opening the dura, small hemorrhages were managed with saline irrigation or pressure but not bipolar cautery. Care was taken also to avoid incidental cisterna magna arachnoid tearing.

After the dural opening was completed, the arachnoid of the cisterna magna was incised with a diamond blade. During the arachnoid incision, care was taken to perform a continuous, linear incision with sharp edges, not serrated. The arachnoid was irrigated copiously with warm saline solution while the incision was performed. As an important note, the edges of the arachnoid membrane were not clipped to the underlying dural flap bilaterally. In this way, the corruption or shrinkage of the membrane was prevented. To prevent the arachnoid from drying out and shrinking during surgery, it was irrigated periodically with warm saline solution.

Posterior fossa surgery was performed, and meticulous hemostasis was achieved. After irrigation of the arachnoid, the edges were gently moved to the midline with fine forceps. The arachnoid membrane was sutured with 10.0 Prolene, beginning from the cranial end of the incision (Figure 1). The running-suture technique was performed. Accordingly, after the first surgical knot was made in the cranial end of the arachnoid opening, continuous suturing with 
a 2-mm distance between the stitches was performed without stretching them. After every 3 stitches, the free end of the thread was pulled gently along the suturing axis, and the edges of the arachnoid were closed (pulling the thread after every 3 stitches instead of after every stitch decreases traumatic injury to the arachnoid edges). During the pulling maneuver, the arachnoid was irrigated with warm saline solution. After the arachnoid edges were approximated, the surgical knot was tied (Video 1). Watertight closure of the cisterna magna arachnoid membrane was checked by the Valsalva maneuver, and no CSF leakage was observed (Figures 2 and 3).

\section{The dura was closed using synthetic or galeal graft tissue. Other anatomical layers were} closed accordingly. The bone flap was placed and fixed with sutures, and the wound was closed in a conventional manner.

All procedures were in accordance with the ethical standards of the institutional and/or national research committee and with the 1964 Helsinki declaration and its later amendments or comparable ethical standards.

\section{RESULTS}

Eleven patients ( 6 male, 5 female) with a mean age of $37.58 \pm 20.34$ years were included in the study. The patients presented mostly with headache, dizziness, and vomiting. Four patients had hydrocephaly at baseline. All patients underwent posterior fossa surgery via a midline suboccipital approach. Arachnoid closure with the continuous-running-suture technique was effective in preventing postoperative incisional CSF leakage and CSF fistula in all the patients. Among 4 patients who had preoperative hydrocephalus, 2 patients underwent ventriculoperitoneal shunt surgery. Seven patients had neither preoperative nor postoperative 
hydrocephalus. Postoperative histopathological investigations revealed mostly glial tumors

(Table 1). No patient suffered from any related complications at the 2- and 6-month postoperative follow-ups.

\section{DISCUSSION}

In the past literature, there are few data available regarding the safety and efficacy of arachnoid suturing in the prevention of CSF leakage after cranial or spinal tumor surgery are available. Previously, Raimondi ${ }^{8}$ recommended close-interrupted or continuous suturing of the arachnoid membrane after spinal surgery, especially after that performed for syringomyelia. More recently, Kim et al. ${ }^{9}$ evaluated the presence of postoperative subdural CSF collection in 88 patients who underwent extracranial-intracranial bypass surgery with (n $=32)$ or without $(\mathrm{n}=51)$ arachnoid membrane suturing. The authors reported that arachnoid suturing significantly decreased the rate of CSF leakage into the subdural space. In addition, Pitskhelauri et al. ${ }^{3}$ investigated pseudomeningocele and incisional CSF leakage rates after cisterna magna surgery with arachnoid membrane suturing followed by sealing with fibrin adhesive material. The study enrolled 70 patients, 32 of whom were in the control group. Postoperative pseudomeningocele and incisional CSF leakage were reported to be seen in $34.4 \%$ and $6.3 \%$ cases, respectively, in the control group. However, these complications were seen in only $5.2 \%$ of the cases in the study group in which the arachnoid membrane was sutured. Accordingly, this technique was found to create an additional barrier for CSF leakage and decreased the risk of postoperative CSF-related complications. The authors performed continuous uninterrupted suturing with 7-0 suture. After completing all sutures without stretching the arachnoid membrane, the free end of the thread was pulled once along the suture to close the incised edges of arachnoid. ${ }^{3}$ However, according to our clinical experiences, we believe that 6-0 or 7-0 sutures might injure the arachnoid membrane. Also, 
pulling the thread only at the end of suturing might cause arachnoid stretching and microtears. Therefore, in our cases, we used 10-0 suture material and the running-suturing technique.

In our study, while opening the dura and separating and peeling away the dura from the arachnoid, we paid attention to avoid inadvertent arachnoid injury. Suturing of the arachnoid membrane is simple for experienced neurosurgeons who are skilled in microanastomosis.

However, a key to success with this type of closure is not only the suturing of the arachnoid but also the cutting. We performed a single, continuous, and sharp incision of the arachnoid membrane. At the end of surgery, we performed arachnoid suturing not only continuously but also with the running-suture technique and the membrane copiously irrigated with warm saline solution. These factors might minimize intraoperative trauma to the arachnoid membrane so that watertight closure and a stable barrier against CSF leakage could be achieved without necessitating supportive fibrin-adhesive material sealing.

There are several limitations in our study. The study had a retrospective case-series design, there was a limited number of patients, and no control group. Still, we believe that the technique described in our study may provide additional information about the efficacy of arachnoid suturing.

According to our study results, arachnoid membrane suturing seems to be safe and effective in preventing postoperative CSF leakage and CSF-related complications. In addition, using the continuous-running-suture technique alone, without any sealant, might be effective in patients with untraumatized arachnoid membrane. Considering the high risk of postoperative CSF leakage and related complications after posterior fossa surgery, we suggest that the 
152 continuous-running-suture technique for closing the arachnoid membrane is worthy of

153 widespread use.

154

155 ACKNOWLEDGEMENT

156 The authors sincerely appreciate the support of the Stead Family Endowed Chair in creation

157 of this work. 


\section{REFERENCES}

1. Kumar A, Maartens NF, Kaye AH. Evaluation of the use of BioGlue in neurosurgical procedures. J Clin Neurosci. 2003;10(6):661-664.

2. Kinaci A, Algra A, Heuts S, O'Donnell D, van der Zwan A, van Doormaal T. Effectiveness of dural sealants in prevention of cerebrospinal fluid leakage after craniotomy: a systematic review. World Neurosurg. 2018;118:368-376.e1.

3. Pitskhelauri D, Kudieva E, Moshchev D, et al. Cisterna magna arachnoid membrane suturing decreases incidence of pseudomeningocele formation and incisional CSF leakage. Acta Neurochir (Wien). 2018;160(5):1079-1087.

4. Culley DJ, Berger MS, Shaw D, Geyer R. An analysis of factors determining the need for ventriculoperitoneal shunts after posterior fossa tumor surgery in children. Neurosurgery. 1994;34(3):402-407; discussion 407-408.

5. Cosgrove GR, Delashaw JB, Grotenhuis JA, et al. Safety and efficacy of a novel polyethylene glycol hydrogel sealant for watertight dural repair. J Neurosurg. 2007;106(1):52-58.

6. Knopp U, Christmann F, Reusche E, Sepehrnia A. A new collagen biomatrix of equine origin versus a cadaveric dura graft for the repair of dural defects - a comparative animal experimental study. Acta Neurochir (Wien). 2005;147(8):877-887.

7. Fang Z, Tian R, Jia Y-T, Xu T-T, Liu Y. Treatment of cerebrospinal fluid leak after spine surgery. Chin J Traumatol. 2017;20(2):81-83.

8. Raimondi AJ. Pediatric Neurosurgery: Theoretical Principles-Art of Surgical Techniques. New York, NY: Springer; 1998:138-139.

9. Kim GW, Joo SP, Kim TS, et al. Arachnoid membrane suturing for prevention of subdural fluid collection in extracranial-intracranial bypass surgery. J Cerebrovasc 
Endovasc Neurosurg. 2014;16(2):71-77. 
184 Figure 1. The arachnoid membrane is closed with 10.0 Prolene suture using the continuous185 running-suture technique. 
186 Figure 2. Illustration of the watertight closure of the arachnoid membrane at the end of 187 surgery.

188 
189 Figure 3. Intraoperative images of watertight closure of the arachnoid membrane during (A)

190 and at the end of (B) suturing. 
191 Video 1. Arachnoid membrane suturing with the continuous-running-suture technique. 
Table 1. Patient demographic and clinical data

\begin{tabular}{lc}
\hline Patient Characteristic & Value \\
\hline Sex (no.) & 6 \\
Male & 5 \\
Female & $37.58 \pm 20.34$ \\
Age (mean \pm SD) (yrs) & \\
Symptoms (no. [\%]) & $9(81.8)$ \\
Headache & $5(45.5)$ \\
Dizziness & $4(36.4)$ \\
Vomiting & $3(27.3)$ \\
Nausea & $3(27.3)$ \\
Ataxia & $4(36.4)$ \\
Preoperative hydrocephaly (no. [\%]) & $0(0.0)$ \\
Preoperative radiotherapy (no. [\%]) & \\
Histopathological diagnosis (no. [\%]) & $3(27.3)$ \\
Metastases & $2(18.2)$ \\
Lung cancer & $1(9.1)$ \\
Breast cancer & $6(54.5)$ \\
Glial tumors & $2(18.2)$ \\
Astrocytoma & $2(18.2)$ \\
Medulloblastoma & $2(18.2)$ \\
Ependymoma & \\
Other & $2(18.2)$ \\
Hemangioblastoma (no. [\%]) & \\
\hline
\end{tabular}




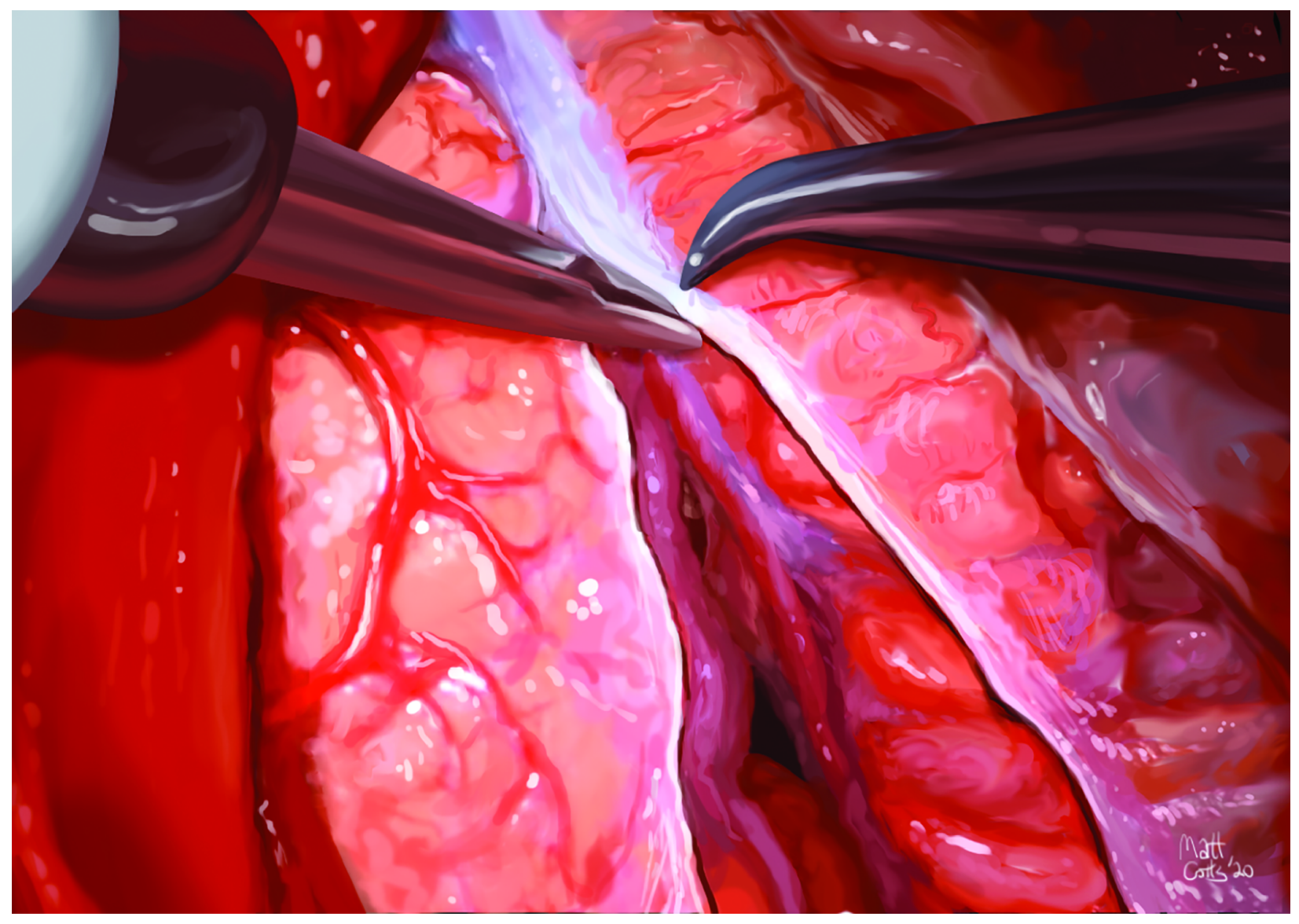




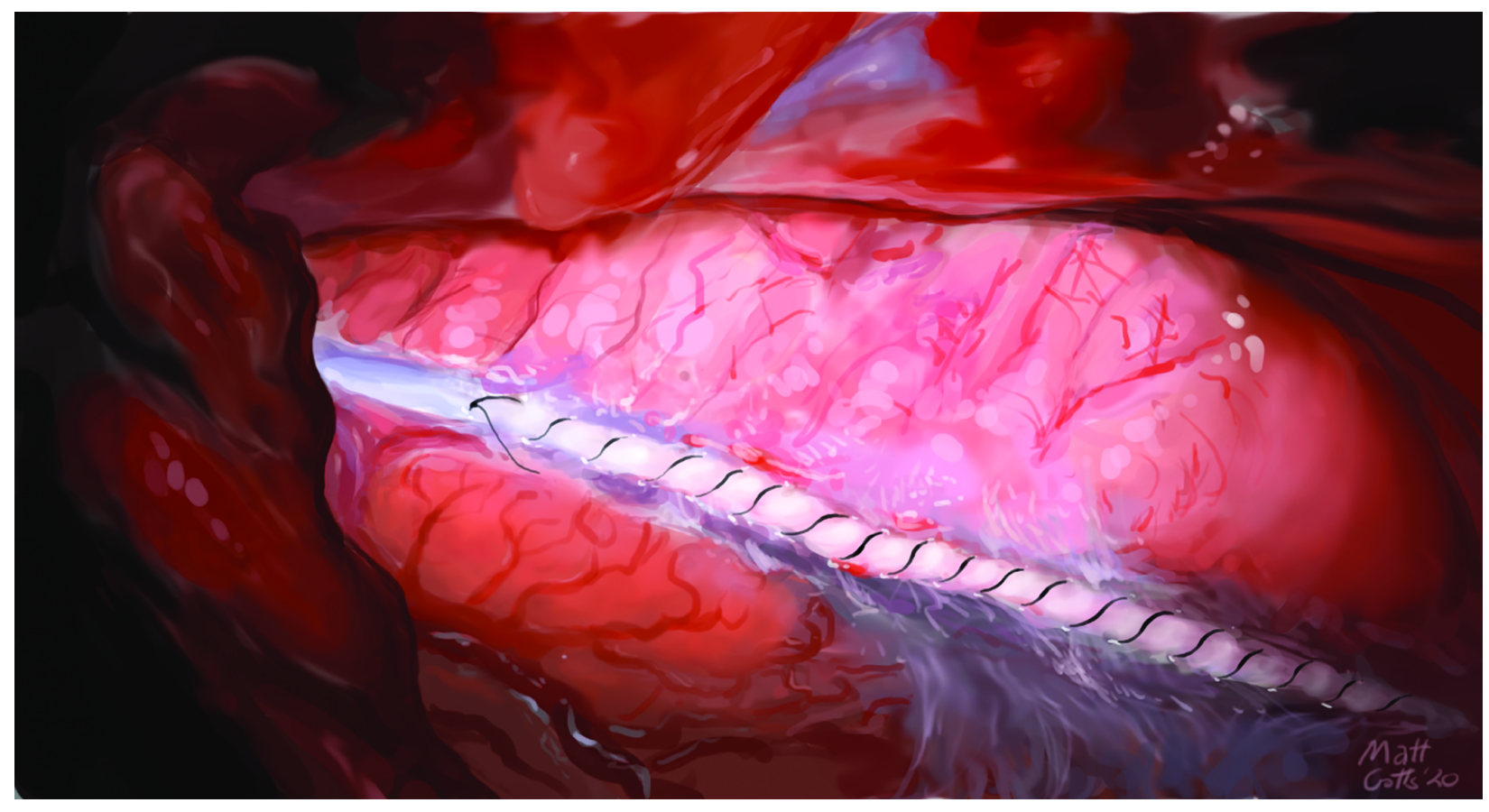




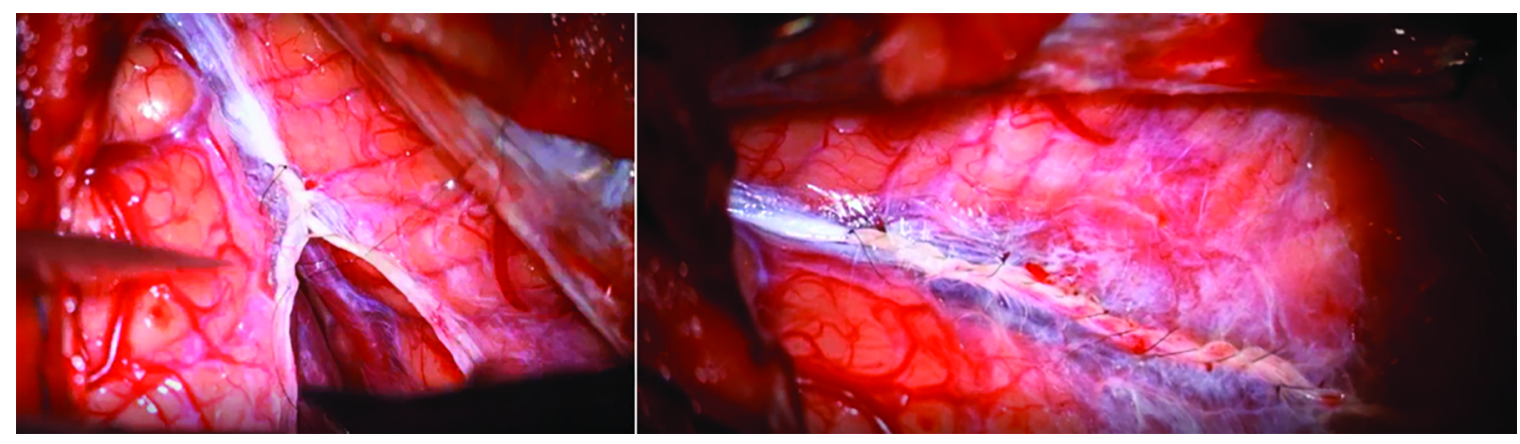


Suturing of the Arachnoid Membrane for Reconstruction of the Cisterna Magna:

Technical Considerations

Abbreviations: none 